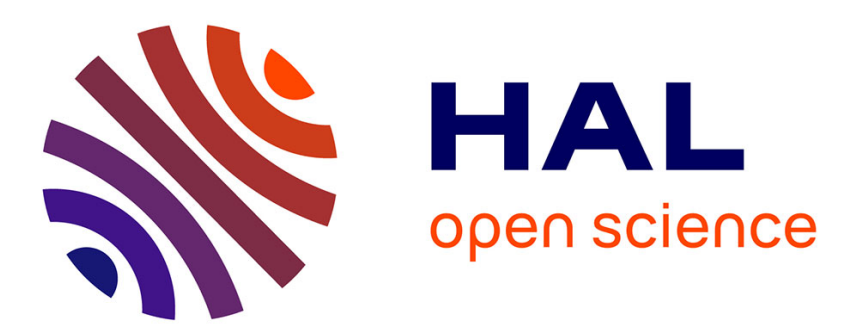

\title{
Experimental study of blade thickness effects on the global and local performances of a Controlled Vortex Designed axial-flow fan
}

\author{
Christophe Sarraf, Hussain Nouri, Florent Ravelet, Farid Bakir
}

\section{To cite this version:}

Christophe Sarraf, Hussain Nouri, Florent Ravelet, Farid Bakir. Experimental study of blade thickness effects on the global and local performances of a Controlled Vortex Designed axial-flow fan. Experimental Thermal and Fluid Science, 2011, 35 (4), pp.684-693. 10.1016/j.expthermflusci.2011.01.002 . hal-00507786v2

\author{
HAL Id: hal-00507786 \\ https://hal.science/hal-00507786v2
}

Submitted on 25 Nov 2010

HAL is a multi-disciplinary open access archive for the deposit and dissemination of scientific research documents, whether they are published or not. The documents may come from teaching and research institutions in France or abroad, or from public or private research centers.
L'archive ouverte pluridisciplinaire HAL, est destinée au dépôt et à la diffusion de documents scientifiques de niveau recherche, publiés ou non, émanant des établissements d'enseignement et de recherche français ou étrangers, des laboratoires publics ou privés. 


\title{
Experimental study of blade thickness effects on the overall and local performances of a Controlled Vortex Designed axial-flow fan
}

\author{
C. Sarraf ${ }^{\mathrm{a}}$, H. Nouri ${ }^{\mathrm{a}}$, F. Ravelet ${ }^{\mathrm{a}, *}$, F. Bakir ${ }^{\mathrm{a}}$ \\ ${ }^{a}$ Arts et Metiers ParisTech, DynFluid, 151 boulevard de l'Hôpital, 75013 Paris, France.
}

\begin{abstract}
The purpose of this work is to study the effects of blade thickness on the performances of an axial-flow fan. Two fans that differ only in the thickness of their blades were studied. The first fan was designed to be part of the cooling system of an automotive vehicle power unit and has very thin blades. The second fan has much thicker blades compatible with the rotomoulding conception process. The overall performances of the fans were measured in a test bench designed according to the ISO-5801 standard. The curve of aerodynamics characteristics (pressure head versus flow-rate) is slightly steeper for the fan with thick blades, and the nominal point is shifted towards lower flow-rates. The efficiency of the thick blades fan is lower than the efficiency of the fan with thin blades but remains high on a wider flow-rate range. The mean velocity fields downstream of the rotors are very similar at nominal points with less centrifugation for the thick blades fan. Moreover, the thick blades fan maintains an axial exit-flow on a wider range of flow-rates. The main differences concern local properties of the flow: Phase-averaged velocities and wall pressure fluctuations strongly differ at the nominal flow-rates. The total level of fluctuations is lower for the thick blades fan that for the thin blades fan and the spectral decomposition of the wall fluctuations and velocity signals reveal more harmonics for the thick blades fan, with less correlation between the different signals. For this kind of turbomachinery, the use of thick blades could lead to a good compromise between aerodynamic and acoustic performances, on a wider operating range.
\end{abstract}

Keywords: Axial-flow fan, turbomachinery, blades thickness, overall performance, pressure fluctuations, phase-averaged anemometry.

\section{Introduction}

The low-speed axial-flow fans, used for instance in the cooling system of automotive vehicles power units, have very complex three-dimensional geometries that can affect both their overall and acoustical performances. An example of one parameter that dramatically modifies the performances is for instance the blade sweep [1-4]. A second parameter that should affect the performances is the blade thickness. Low-speed axial-flow fans usually have conventional thin blades. However, following motivations such as active control of the blade shape or the use of the rotomoulding process $[5,6]$, the designers would have now to face profiles with thicker blades. The control of blade shape may help to reduce the radiated noise or to extend the operating range and the need for actuators then implies thicker blades. The rotomoulding process previously tested for wind turbine blades - leads to thick hollow blades and may be more economic because of material gain. The blades can have more stiffness with greater inertia moment. Complex shapes with blade recovering are moreover easier to manufacture than with traditional

\footnotetext{
*corresponding author

Email address: florent.ravelet@ensta.org (F. Ravelet)
}

plastic injection methods, and the blades can be filled for instance with noise-absorbing foam.

In aeronautics and in the automotive industry, changing the blade thickness has been used for many years as an efficient way to modify the lift and drag characteristics and the boundary layers detachment process [7, 8]. Applied to low-speed axial-flow fans, one could a priori think that the extra thickness may increase the dissipation of energy in the von Kármán street behind the blades so that the performances of the fan decrease and the pressure fluctuations in its wake increase. The effects of blade thickness on the overall performances are not so well known [9-12] and to the best of our knowledge the influence on the dynamics remains an open question.

Therefore this study intends to compare the aerodynamical performances of two axial-flow fans that only differ in the thickness of their blades. The geometry of the fans and the experimental set-up are presented in $\S 2$. The results on the mean features are presented and discussed in $\S 3$ : the overall characteristics of the two fans are presented in $\S 3.1$; the mean velocity fields downstream of the fans are compared in $\S 3.2$ and these results are briefly discussed in $\S 3.3$. Some results on local fluctuating quantities are presented and discussed in $\S 4$ : measurements of the wall pressure fluctuations that are an image of the 
acoustical performances are presented in $\S 4.1$ and finally, phase-averaged velocity signals that are measured on one point are then compared in $\S 4.2$. Concluding remarks are then given in $\S 5$.

\section{Experimental setup}

\subsection{Geometry of the two fans}
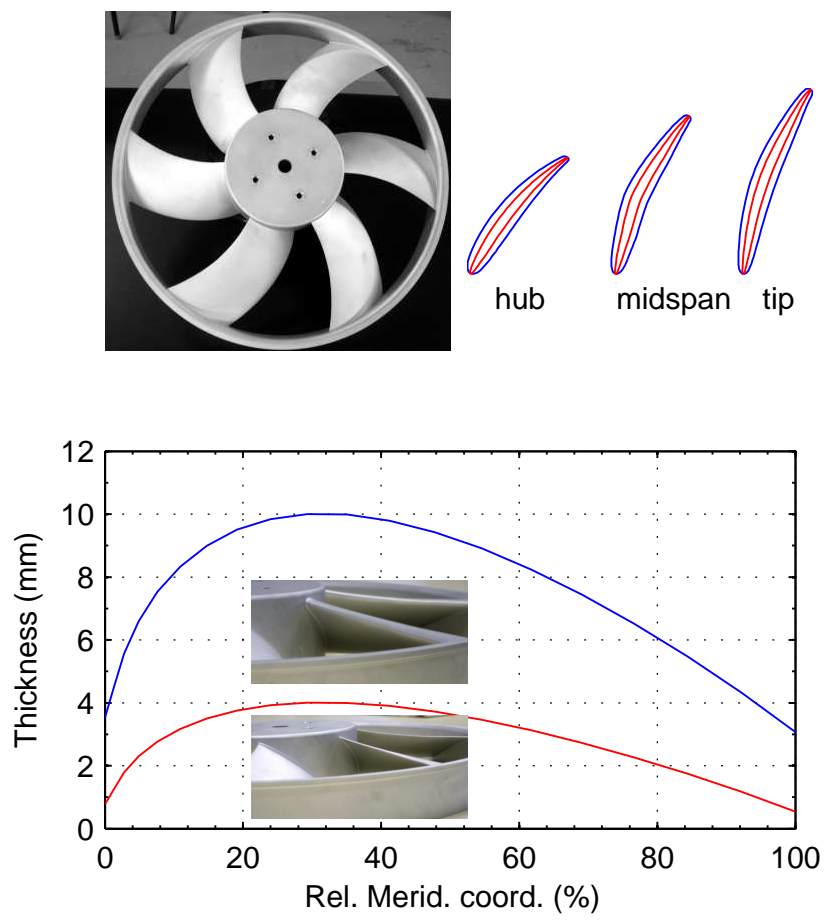

Figure 1: Views of the two fans. Front view, sections of thick and thin profiles at various span locations and thickness distribution law along the meridional coordinate.

The fans used in this study are two prototypes developed for automotive engine cooling system applications following the methods detailed in [13]. The fans are made out of aluminium and have the same geometrical characteristics except the blade thickness. The thin blades axialflow fan is the reference and is labeled $F_{A}$. The thick blades fan is labeled $F_{B}$. Pictures of the thick blades fan are displayed in Fig. 1 with a drawing of a section of the $F_{A}$ and $F_{B}$ blades at hub, mid-span and tip. The main characteristics of the blade cascade are given in Tab. 1.

Each of these fans has six blades. The rotor is built up from blades of circular-arc camber lines, with NACA65 profiles of mean chord $c=74 \mathrm{~mm}$ clipped at $0.95 x / c$. The hub-to-tip radius ratio is $R_{\text {int }} / R_{\max }=0.365$ with tip radius $R_{\max }=179 \mathrm{~mm}$. The rotors are moreover shrouded with a zigzag circular collar in order to close the radial gap in the free-flow experimental facility (see $\S 2.2$ below). The spanwise load of the blades is prescribed following a controlled vortex design method [3, 13]: in the present case the absolute tangential velocity downstream of the rotor $V_{\theta}$ increases linearly with the radius such that the Euler work per unit mass $\omega r V_{\theta}$ increases quadratically from the hub to the tip of the blades. The blade loading that is the ratio of the Euler work to the square of the peripheral velocity is moreover constant along the radial direction. With this design the hub losses are reduced and the blades are less twisted [3]. The blades are stacked on the trailing edge. The blades are finally swept forward in order to benefit from lower tonal noise as suggested by several studies [1-4]. The forward sweep may also increase or decrease the overall performance, depending on the blade loading distribution, as reported in Refs. [3, 4].

The maximum blade thickness of the thin blade axialflow fan $F_{A}$ is $4 \mathrm{~mm}$, i.e. $5.6 \%$ of the chord length at mid-span (conventional axial-flow fans usually have a relative maximum thickness varying from roughly $10 \%$ at hub to $7 \%$ at tip [13]). The blades of the second fan $F_{B}$ are thickened to reach a maximum thickness of $10 \mathrm{~mm}$, i.e. $13.5 \%$ of the chord length at mid-span. The thickness distribution $e$ of fan $F_{B}$ is: $e_{B}=2 e_{A}+2 \mathrm{~mm}$. The Fan $F_{A}$ was designed to meet the specifications point $\Delta p_{\text {des }}=$ $270 \mathrm{~Pa}, Q_{N}$, des $=0.736 \mathrm{~m}^{3} . \mathrm{s}^{-1}$ for a nominal angular velocity $\omega_{N}=260$ rad.s ${ }^{-1}$. The corresponding specific speed $\Omega=\frac{\omega \sqrt{Q}}{(\Delta p / \rho)^{3 / 4}}$ is $\Omega=3.89$.

\subsection{Normalized test benches and measurement techniques} Free-flow experimental facility

The Figure 2a shows the experimental facility used to determine the overall performances of axial-flow fans in open-flow configuration. The air suction test bench was designed and built at the Dynfluid laboratory of Arts et Métiers ParisTech according to the ISO-5801 standard. It consists of a cuboid of size $1.3 \times 1.3 \times 1.8 \mathrm{~m}$. A pierced plate inside it prevents the flow from having prerotation. The fans are placed at one end, the zigzag circular collar being tightly fitted to the bench. The air flow-rate is set and measured according to the ISO-5167 standard by setting the hydraulic impedance of the bench through diaphragms of various sizes that are placed at the other end.

Pressure rise $\Delta p$ is measured with an absolute precision of $\pm 0.1 \mathrm{~Pa}$. The torque $C$ on the fan shaft is measured with a HBM strain gauge transducer. The uncertainty of torque measurements corresponds to $0.1 \%$ of the maximum measured torque. The angular velocity $\omega$ is measured with a tachymeter of relative precision $\pm 0.2 \%$. The power adsorbed on the fan shaft is then estimated through the product $C \omega$. The accuracy of the whole system leads to a determination of the efficiency within about $\pm 0.5 \%$.

The mean velocity field in the wake of the fans has been measured with Two-Component Laser Doppler Anemometry (LDA), using a DANTEC "FlowExplorer" system. The method to get the three components of the mean velocity field downstream of the fan relies on the axisymmetry of the mean flow, that is a posteriori checked. The definition of the laboratory Cartesian coordinate system $\{X ; Y ; Z\}$ and of the two measuring planes is pictured in Fig. 3. The LDA system measures the velocities on the 


\begin{tabular}{c|c|c|c|c|c|c}
\hline \hline & $R$ & $c$ & $\sigma$ & $\gamma$ & profile & $D$ \\
\hline Hub & 65.4 & 66.6 & 0.97 & 53 & NACA 65(07)06 & 0.372 \\
\hline Mid-span & 122.2 & 74.0 & 0.58 & 66 & NACA 65(10)05 & \\
\hline Tip & 179.0 & 81.3 & 0.43 & 70 & NACA 65(11)05 & 0.650 \\
\hline \hline
\end{tabular}

Table 1: Blade cascade parameters for the reference fan $F_{A}$. Radius $R(\mathrm{~mm})$. Chord length $c$ (mm). Cascade solidity $\sigma$. Stagger angle $\gamma$ $\left({ }^{\circ}\right)$. Profile designation according to the nomenclature given in Ref. [13]: NACA65(xx)yy with (xx) representing the relative camber and yy standing for the relative thickness. Lieblein's diffusion factor $D$.

$Y$ axis and on the $Z$ axis. The measurement is first performed in the $\{Y ; Z\}$ plane at a certain radial distance $r$ from the axis of rotation, and at an (axial) distance $z$ from the trailing edges of the fan in the $Y$ direction (point 1 in Fig. 3). The measured velocities, in polar coordinates, are thus the radial velocity $V_{r}(r, z)$ and the axial velocity $V_{z}(r, z)$. The measuring volume is then precisely traversed to the same radial and axial distances $r$ and $z$, but in the $\{X ; Y\}$ plane (point 2 in Fig. 3). Special care has been given to the alignment of the system. The velocities that are measured at this second point, in polar coordinates, are thus the opposite of the tangential velocity $-V_{\theta}(r, z)$ and the axial velocity $V_{z}(r, z)$ that is measured two times. We then check that the two measurements give the same mean axial velocities: the difference between $V_{z}(r, z)$ in the two planes is less than $1 \%$ everywhere.

Constant Temperature Anemometry (CTA) with a 2D Dantec 55R51 hot fiber film probe has also been used to study more specifically the velocity fluctuations and the phase-averaged velocity signals. The method to get the three velocity components is the same as described in Refs. [2, 4] and is based on two measurements at different angular positions of the probe.

\section{Ducted-flow experimental facility}

In order to compare the behavior of the two fans in a ducted-flow configuration and to measure the wall pressure fluctuations, a second test bench was built according to the ISO-5801 standard (see Fig. 2b).

It consists of a cylindrical pipe of inner diameter $D=$ $380 \mathrm{~mm}$. A bell mouth is flush-mounted at the inlet of the duct. The upstream face of the fan is at a distance $D$ from the pipe inlet. A DC-motor is hidden in a casing of diameter $0.3 \mathrm{D}$ and length $0.7 \mathrm{D}$, with a warheadshape end. The binding to the tube is ensured by five rods of diameter $8 \mathrm{~mm}$, i.e. $0.02 \mathrm{D}$, in order to minimize their influence, regardless the flow-rate. The distance between the upstream face of the fan and the binding rods is $0.26 D$. An anti-gyration device made of eight metal sheets of thickness $1.5 \mathrm{~mm}$ and length $2 D$ is placed $2 D$ downstream of the rotor-stator set. It prevents the outgoing flow from having any rotating component. The static pressure of the axial fan is measured $1 D$ downstream of the anti-gyration device, with an average over four flushmounted pressure taps. The flow-rate is measured with a normalized diaphragm, located $10 D$ downstream of the anti-gyration device and $5 D$ upstream of the pipe outlet. The diaphragm has a diameter of $0.73 D$. Other diaphragms of various sizes are placed at the exit of the pipe to vary the test-bench hydraulic impedance and thereby to vary the operating point of the studied axial-flow fan.

Wall pressure fluctuations are measured simultaneously by eight microphones that are evenly distributed on the circumference of the duct. They are mounted downstream of the fan, halfway between the fan and the five rods that ensure the binding to the tube (see Fig. 2b). The microphones are G.R.A.S 40BP 1/4" polarized pressure microphones of sensitivity 1.65 mv.Pa ${ }^{-1}$, with G.R.A.S 26AC preamplifiers and a G.R.A.S $12 \mathrm{AG}$ power supply module. The signals are amplified with a gain of $+30 \mathrm{~dB}$ and highpass filtered with a three-pole Butterworth filter with cutoff frequency of $20 \mathrm{~Hz}$. The signals are then digitalized using a NI Data Acquisition Card (M 6211, 16 bits) at a sample rate of $12 \mathrm{kHz}$.

\section{Comparison of the mean features of the two fans}

\subsection{Overall characteristics}

The characteristics of the two fans are shown in Fig. 4a together with the design operation point of the reference fan. The flow-rate coefficient $(\Phi)$ and pressure rise coefficient $(\Psi)$ are defined respectively by equations 1 and 2 :

$$
\begin{aligned}
\Phi & =\frac{Q}{\pi \omega R_{\max }^{3}} \\
\Psi & =\frac{\Delta p}{\frac{1}{2} \rho \omega^{2} R_{\max }^{2}}
\end{aligned}
$$

The design point thus corresponds to $\Phi_{\text {des }}=0.156$ and $\Psi_{d e s}=0.200$ and is indicated by a dot in Fig. 4a.

The curves are the results of five measurements at the nominal rotation rate $\omega_{N}=260 \mathrm{rad} . \mathrm{s}^{-1}$. The error bars in Fig. 4a stand for the standard deviation of the five measurements of $\Phi$ and $\Psi$, magnified by a factor three for better visualization. The pressure coefficient distributions are relatively similar to each other. The thin blades fan $F_{A}$ matches the specifications point plotted in Fig. 4. At the design flow-rate, the thick blades fan $F_{B}$ produces a pressure rise that is $8 \%$ below the specified pressure rise. The curve of $F_{B}$ is moreover steeper than the curve of $F_{A}$ and there is a cross-over between the two fans: at partial flow-rates below $\Phi=0.11, F_{B}$ produces more pressure rise 
(a)

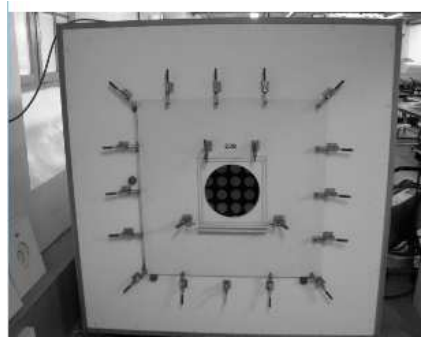

(b)
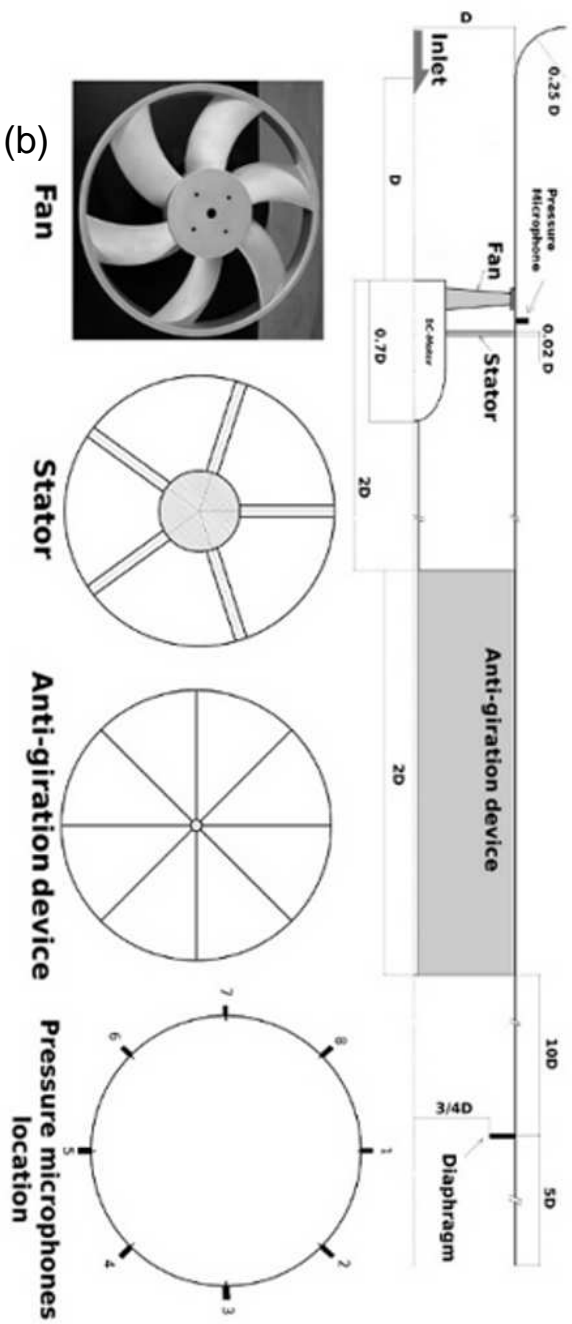

Figure 2: ISO-5801 test benches. (a): open-flow facility. The dimensions are $1.3 \times 1.3 \times 1.8 \mathrm{~m}$. The fans suck the flow through the test bench. (b): ducted-flow facility. This configuration is dedicated to the local measurements of wall pressure fluctuations. The fans blow the flow into the pipe.

than $F_{A}$; at overflow-rates, approximately beyond $\Phi=0.2$, $F_{A}$ produces this time more pressure rise than $F_{B}$ and the difference of pressure rise between the two fans remains almost constant.

The efficiency coefficient $(\eta)$ is defined by equation 3 :

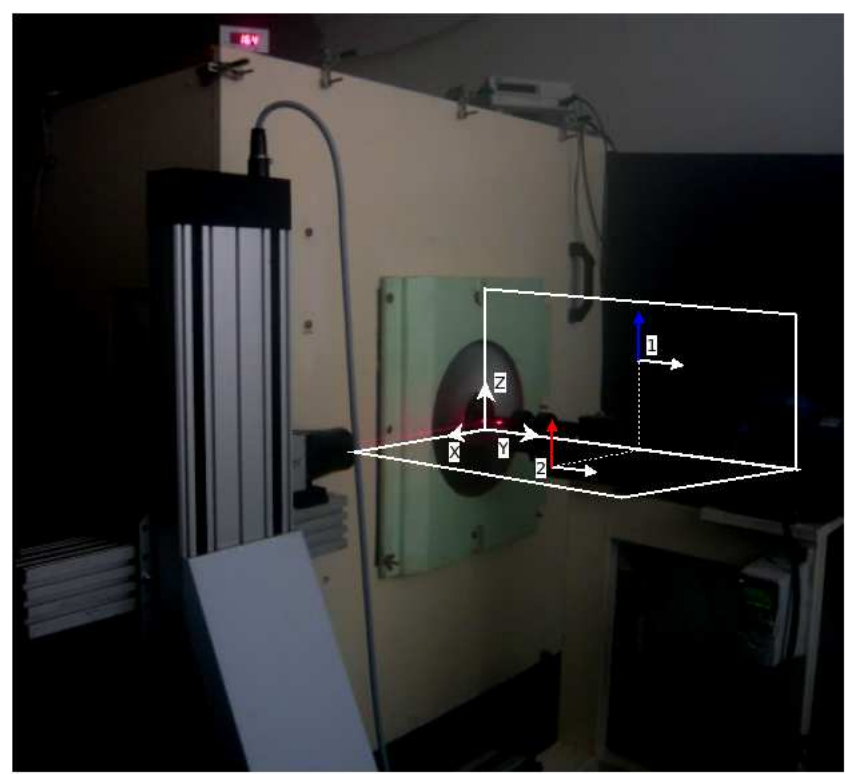

Figure 3: Measurement of the mean exit-flow field with a Twocomponent LDA system. The measurements are taken in the two planes that are displayed with white lines. The origin of the coordinate system is centered on the rotation axis, and the axial position of the origin corresponds to the plane that contains the fans blades trailing edges. The LDA system measures the velocity in the $Y$ and $Z$ directions. At point 1 this corresponds to the axial $V_{z}$ and radial velocities $V_{r}$. At point 2 this corresponds to the axial $V_{z}$ and to the opposite of the tangential velocities $-V_{\theta}$

$$
\eta=\frac{\Delta p Q}{C \omega}
$$

The efficiencies of both fans are shown in Fig. 4b with the flow-rate coefficient of the design point highlighted by the vertical dash-dotted line. The efficiencies distributions have a classical shape, with a slow evolution for partial flow-rates and a strong drop after the nominal flow-rate. The nominal flow-rate for $F_{A}$ is $\Phi_{N, A}=0.18$ and the corresponding pressure coefficient is $\Psi_{N, A}=0.17$. The efficiency is then $\eta_{\max , A}=0.58 \pm 0.005$ which is pretty fair for this type of turbomachinery [13]. The maximum of $\eta$ is shifted towards overflow with respect to the design point. This point is discussed below in $\S 3.3$.

The maximum efficiency for the thick blades fan $F_{B}$ is $\eta_{\max , B}=0.55 \pm 0.005$. The thickening of the blades has led to a decrease of 0.03 of $\eta_{\max }$. The nominal flowrate of $F_{B}$ is shifted toward a partial flow-rate of about $17 \%$ with respect to the nominal flow-rate of $F_{A}$ and the thick blades fan thus gives its best efficiency for a flow-rate $\Phi_{N, B}=0.15$ very close to the design point $\Phi_{\text {des }}=0.156$. The corresponding pressure coefficient is $\Psi_{N, B}=0.19$. The distribution of the efficiency of $F_{B}$ is moreover flatter than that of $F_{A}$ : quantitatively, the range of flow-rates corresponding to a decrease of $3 \%$ of $\eta$ from $\eta_{\max }$ is \pm 0.3 $\Phi / \Phi_{N}$ for $F_{B}$, while it is only $\pm 0.2 \Phi / \Phi_{N}$ for $F_{A}$.

As a partial conclusion please also note that although the thick blades fan $F_{B}$ does not meet the specification 

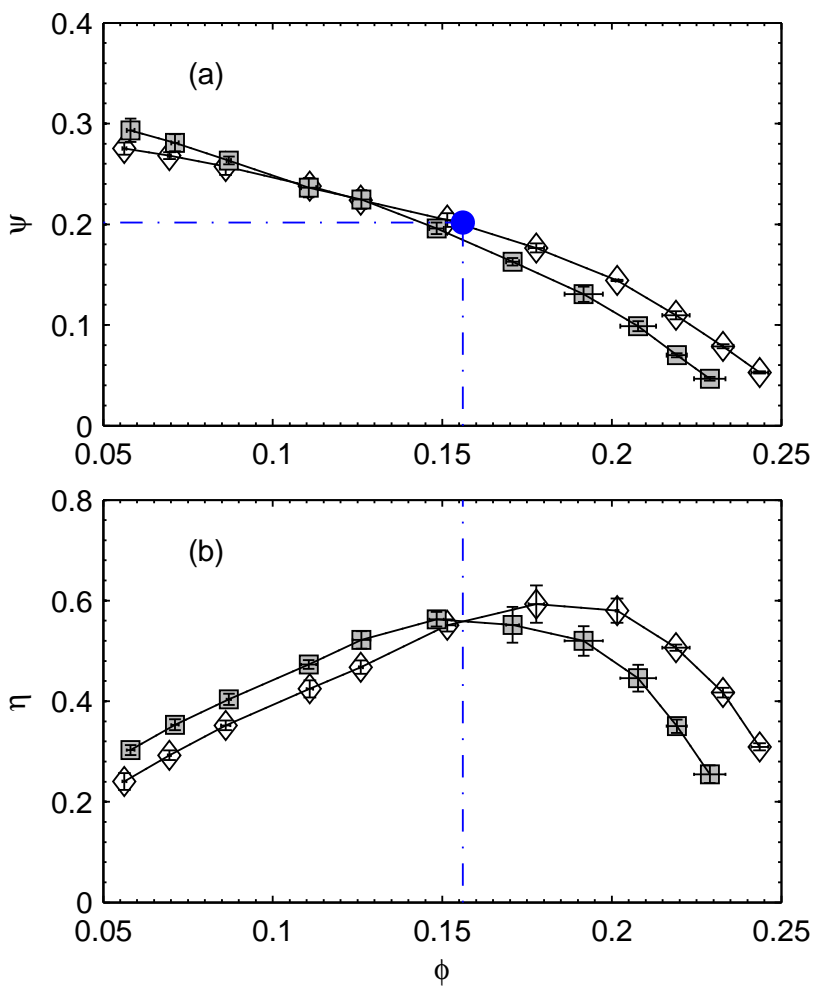

Figure 4: Fans characteristics: (a) Pressure rise coefficient $\Psi$ vs. flow-rate coefficient $\Phi$ and (b) Fans efficiency $\eta v s$. flow-rate coefficient $\Phi .(\diamond)$ : thin blades fan $F_{A},(\square)$ : thick blades fan $F_{B} \cdot(\bullet)$ : design specification point. The reproducibility is evaluated from 5 independent experiments and displayed are errorbars magnified by a factor 3 for (a) and a factor 6 for (b)

point at the nominal angular velocity $\omega_{N}=260 \mathrm{rad} . \mathrm{s}^{-1}$, the desired point in a dimensional point of view $(\Delta p=$ $\left.270 \mathrm{~Pa}, Q_{N}=0.736 \mathrm{~m}^{3} . \mathrm{s}^{-1}\right)$ can be reached with this fan rotating at $\omega=270 \mathrm{rad} . \mathrm{s}^{-1}$. Since the efficiency of the two fans is the same at such flow-rates $(\eta \simeq 0.55)$, the use of $F_{B}$ at $\omega=270$ rad.s ${ }^{-1}$ in the automotive cooling application would lead to the very same power consumption as the use of $F_{A}$ at $\omega_{N}=260 \mathrm{rad} . \mathrm{s}^{-1}$.

\subsection{Comparison of the mean exit-flow fields}

The mean exit-flow downstream of the rotors may give further information that help to understand the differences that have been observed on the overall characteristics. To compare the two fans $F_{A}$ and $F_{B}$, two sets of measurement of the flow field have been performed. Each set consists in choosing one dimensional operating point (fixed diaphragm at the inlet of the free-flow experimental facility, flow-rate $Q$ and pressure rise $\Delta p$ ) and in adjusting the rotation rates of the two fans to make them operating at this very same point.

The first set was made very close to the nominal flowrate of $F_{B}\left(\Phi_{N, B}=0.15\right)$ and is presented in Fig. 5 . The second set was made very close to the nominal flow-rate of $F_{A}\left(\Phi_{N, A}=0.18\right)$ and is presented in Fig. 6. The flow fields have been measured on a Cartesian grid that extends typically from $z=20 \mathrm{~mm}$ to $z=150 \mathrm{~mm}$ with a spacing of $10 \mathrm{~mm}$ (14 points in the axial direction) and from $r=40 \mathrm{~mm}$ to $r=220 \mathrm{~mm}$ or from $r=65 \mathrm{~mm}$ to $r=255 \mathrm{~mm}$ with a spacing of $5 \mathrm{~mm}$ (38 points in the radial direction). The mean data rate is $2000 \mathrm{~Hz}$ and the signal has been acquired for $15 \mathrm{~s}$, i.e. roughly 500 revolutions of the rotor, at each position. The mean value and the standard deviation are thus well converged.

\section{Discharge flow components}

The flow fields in meridional plane, $i$.e. the discharging component $V_{z}(r, z)$ and the centrifugal component $V_{r}(r, z)$, are displayed in Figs. 5a-b and 6a-b.

One common feature of the two fans, regardless of the flow-rate, is the presence of a large recirculation, with small upstream velocities close to the hub: the discharged flow exits only for $r>100 \mathrm{~mm}$. Measurements of the flow field close to the hub with CTA that do not give access to the velocity direction should be considered with caution. The main difference lies in the centrifugation of the flow by the two fans.

The meridional flow for $F_{A}$ at a partial flow-rate of $\Phi / \Phi_{N, A}=83 \%$ is plotted in Fig. 5a. The flow downstream of the fan already has a very important radial component as noticed by Kergoulay et al. [2]. The discharging flows for the two fans at their respective nominal flowrate (Fig. 6a for $F_{A}$ and Fig. $5 \mathrm{~b}$ for $F_{B}$ ) are very similar with smaller centrifugation for $F_{B}$. The meridional flow for $F_{B}$ at an overflow-rate of $\Phi / \Phi_{N, B}=120 \%$ is plotted in Fig. 6b and presents barely no centrifugation. There thus seems to be a tendency for the thick blades fan $F_{B}$ to maintain a mainly axial exit-flow on a wider range of operating points.

\section{Mean tangential velocity}

Contours of the tangential velocity field $V_{\theta}(r, z)$ are plotted in Figs. 5c-d and 6c-d. As a common feature for both fans, one can notice that the tangential velocity downstream of the rotors has significant values, of the order of the discharge velocity $Q /\left(\pi R_{\max }^{2}\right)$. At the design flow-rate, an extra static pressure rise of roughly $1 / 2 \rho V_{\theta}^{2}=$ $30 \mathrm{~Pa}$ could be obtained by the use of a stator. That corresponds to $20 \%$ of the pressure rise and would lead to a significant increase of the efficiency. The tangential velocity fields are moreover strongly correlated to the meridional velocity field for both fans, regardless of the flow-rate. It is almost zero outside the discharge jet. The $3 D$ structure of the exit-flow thus resembles an annular rotating jet. Close to the fans, there are two local maxima for nominal (Figs. 5d for $F_{B}$ and $6 \mathrm{c}$ for $F_{A}$ ) and overflow-rates (Fig. 6(d) for $F_{B}$ ). The radial positions of these maxima are adjacent to the edges of the discharge jets.

The differences between the two fans lie in the magnitude and the axial attenuation of the tangential velocities. The velocities are stronger for the thick blades fan $F_{B}$ which is consistent with its overall lower efficiency - and 
the axial velocity decay is also slower. These two points are discussed below in $\S 3.3$.

\section{Standard deviation of the tangential velocity}

Contours of the standard deviation (or the root mean square $r m s$ ) of the tangential velocity field $V_{\theta} r m s$ are plotted in Figs. 5e-f and 6e-f. The maxima of fluctuations are located at the edge of the discharge jet where the radial gradient in tangential velocity is steep, and the core of the jet corresponds to a local minimum of fluctuations. Finally, one can notice that the global level of fluctuations in the exit-flow of $F_{A}$ is stronger than that in the exit-flow of $F_{B}$.

\subsection{Remarks and discussion on the modifications of over- all performances and of exit-flow topology}

The nominal flow-rates of the two fans are different and furthermore do not match the design point (see Fig. 4b). The latter could be an effect of the forward sweep as noticed in Refs. [3, 4] : for comparable Controlled Vortex Design fans — of relative thickness $10 \%$ and pressure rise coefficient $\Psi_{N} \simeq 0.3$ for $\operatorname{Vad}[3]$ and of relative thickness $9 \%$ and pressure rise coefficient $\Psi_{N} \simeq 0.3$ for Hurault $e$ t al. [4] — both studies reported a decrease of the flow-rate and efficiency with respect to the design point when the fan blades are swept forward. However, the trend is not systematic and first depends on the blade loading that is directly linked to half of the pressure rise coefficient $\Psi_{N}$. Indeed, for a more loaded fan with more load near the tip, Hurault et al. [14] reported an increase of the nominal flow-rate with forward sweep. The fact that the nominal flow-rates of $F_{A}$ is greater than the design point computed with a method that had been validated for conventional thickness of roughly $7 \%$ [13] — while it is lower for $F_{B}$ in the present study suggests that the shift of nominal flow-rate with respect to the design point could not be ascribed to the blade sweep alone but that blade thickness also plays a major role because it somehow modifies the blade loading and the balance between lift and losses.

Some results concerning the effects of thickness on the aerodynamic behavior of isolated profiles may help to explain the relative position, i.e. the cross-over between the two curves (see Fig. 4a). In general the thickening of isolated profiles leads to a reduction of the aerodynamic efficiency (lift to drag ratio) [7, 8]. Even at the most efficient angle of attack, significant boundary layer separations are observed on thick profiles - of relative thickness above $10 \%$ - while not on thin profiles [11] and the lift is reduced. The viscous dissipation is moreover greater for thick profiles, especially when they have rounded trailing edge as it is the case here [10]. Despite these drawbacks, the thick profiles have the advantage of having a more progressive stall than thin profiles, at higher angles of attack. The first two points are most likely responsible for the lower pressure rise and the lower efficiency of $F_{B}$ at nominal and overflow-rates where the angle of attack of the blade cascade is low: at high flow-rates the lift is increased for the thin blades fan $F_{A}$ that means more pressure rise and the viscous losses are reduced and this could explain the shift of the nominal point towards higher flow-rates. The last feature is almost probably at the origin of the gain of pressure rise and of efficiency at partial flow-rates as observed for $F_{B}$ in the present experiment: the losses that are dominated by the separation on the suction side are reduced and the lift is increased.

The effects of the thickness on the overall performances close to the nominal flow-rates could also be informed by the modifications of the exit-flow topology (see Figs. 5 and 6 ). The tangential velocities downstream of the fan $F_{B}$ (Fig.5d and Fig. 6d) are stronger than for the fan $F_{A}$ (Fig. 5c and Fig. 6c). Since the velocity fields for the two fans have been measured at the same flow-rate and pressure rise, $F_{B}$ rotating a little bit faster than $F_{A}$ for that, the Euler work for $F_{B}$ is thus greater than the Euler work for $F_{A}$ and its static efficiency is lower. However, if one would have to use a stator, the fan $F_{B}$ would lead to a greater recovery of static pressure which would increase the static efficiency of the stage. The regions of high velocity also extend further away axially and are moreover larger in radius for $F_{B}$ : a stator could be positioned in a wider range of axial distance from the fans, which should be beneficial regarding interaction noise. And at last, the fact that the thick blades fan $F_{B}$ maintains a mainly axial flow on a wider range of flow-rates (less centrifugation) would also increase the efficiency of a stator downstream of it. 

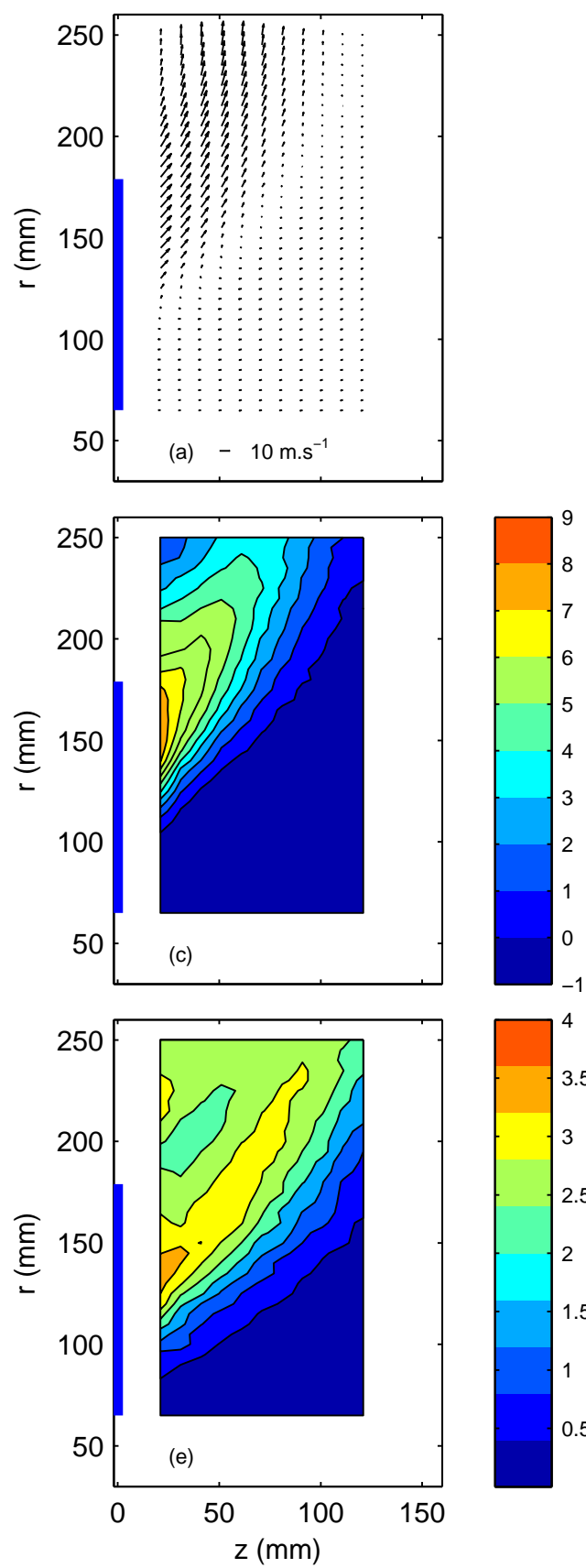
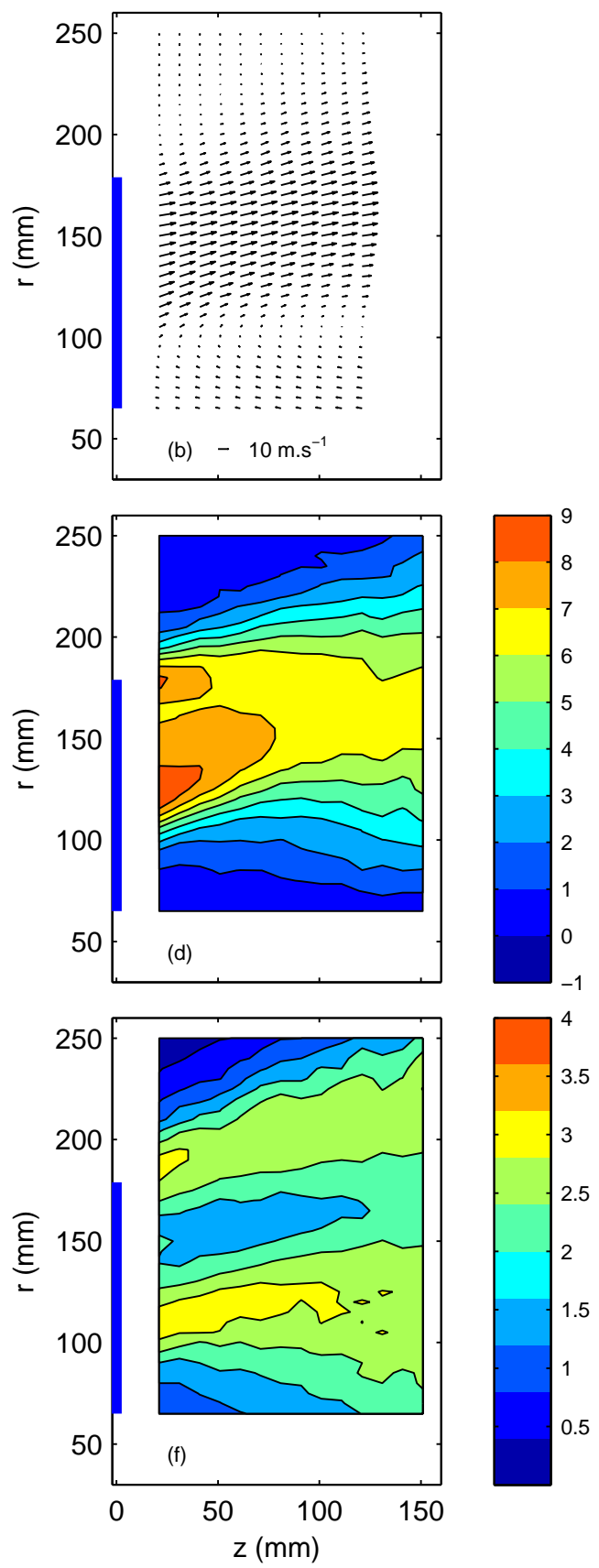

Figure 5: Mean exit-flow measured by LDA close to the nominal point of $F_{B}$. (a-c-e): $F_{A}$ rotating at $\omega=209.5$ rad.s ${ }^{-1}$ for a flow-rate $Q=0.557 \mathrm{~m}^{3} \cdot \mathrm{s}^{-1}$ and a pressure rise $\Delta p=164 \mathrm{~Pa}$ corresponding to a flow coefficient $\Phi_{A}=0.148$. The discharge velocity $Q /\left(\pi R_{m a x}^{2}\right)$ is $5.6 \mathrm{~m} . \mathrm{s}^{-1}$. (b-d-f): $F_{B}$ rotating at $\omega=214.2 \mathrm{rad} . \mathrm{s}^{-1}$ to give the same flow-rate and pressure rise, that corresponds then to a flow coefficient $\Phi_{B}=0.144$. (a-b): Mean meridional flow $\left\{V_{r}(r, z) ; V_{z}(r, z)\right\}$. (c-d): mean tangential velocity $V_{\theta}$. (e-f): Standard deviation of the tangential velocity $V_{\theta} r m s$. The blue patch in each figure stands for the position of the blades 

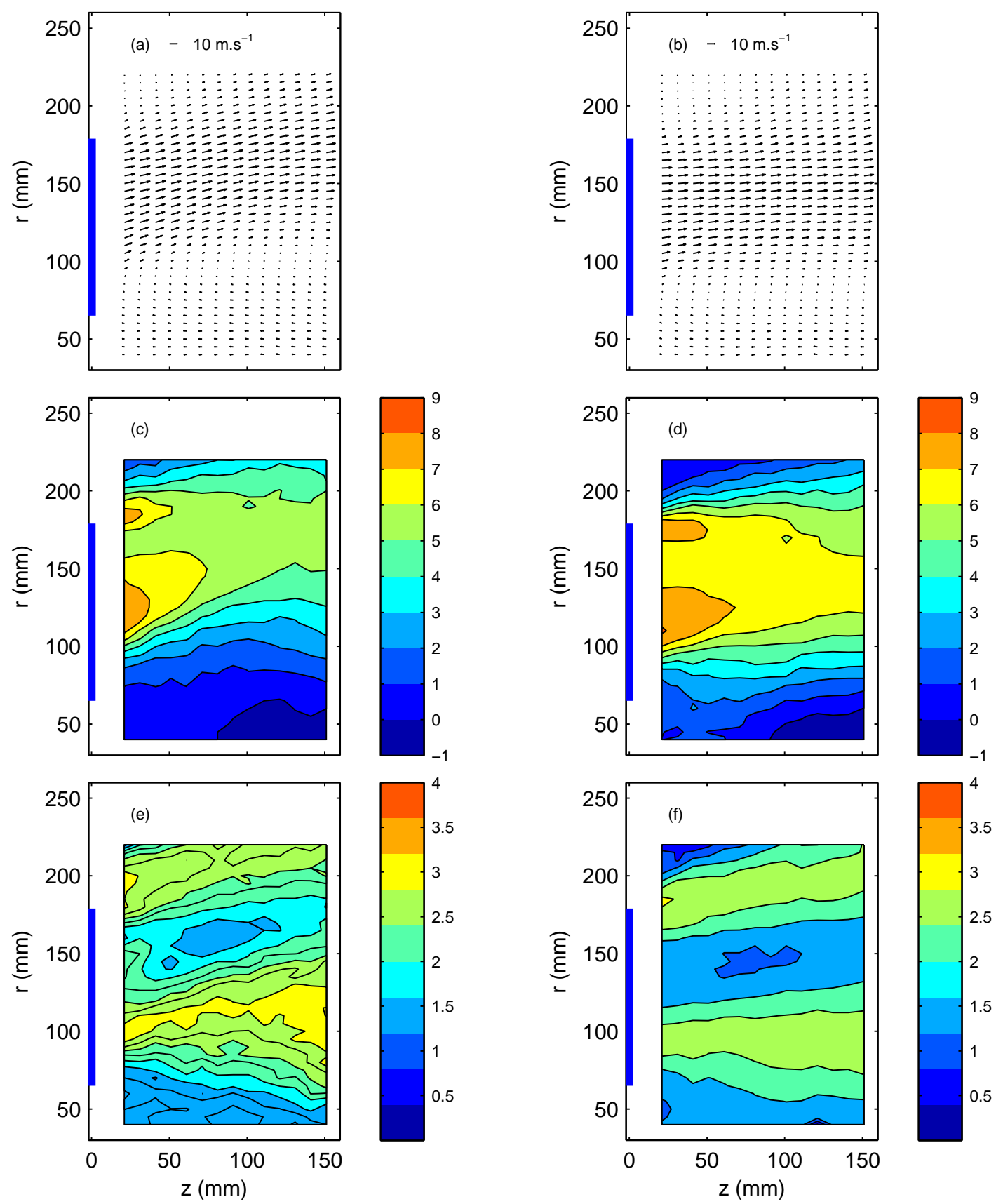

Figure 6: Mean exit-flow measured by LDA close to the nominal point of $F_{A}$. (a-c-e): $F_{A}$ rotating at $\omega=209.5$ rad.s ${ }^{-1}$ for a flow-rate $Q=0.668 \mathrm{~m}^{3} \cdot \mathrm{s}^{-1}$ and a pressure rise $\Delta p=140 \mathrm{~Pa}$ corresponding to a flow coefficient $\Phi_{A}=0.178$. The discharge velocity $Q /\left(\pi R_{m a x}^{2}\right)$ is $6.7 \mathrm{~m} . \mathrm{s}^{-1}$. (b-d-f): $F_{B}$ rotating at $\omega=218.1 \mathrm{rad} . \mathrm{s}^{-1}$ to give the same flow-rate and pressure rise, that corresponds then to a flow coefficient $\Phi_{B}=0.170$. (a-b): Mean meridional flow $\left\{V_{r}(r, z) ; V_{z}(r, z)\right\}$. (c-d): mean tangential velocity $V_{\theta}$. (e-f): Standard deviation of the tangential velocity $V_{\theta} r m s$. The blue patch in each figure stands for the position of the blades 


\section{Study of local fluctuations}

The increase of blade thickness thus leads to strong differences in the mean-flow features of those two fans. The purpose of the present section is to study the influence of the blade thickness on local dynamics such as pressure and velocity fluctuations.

\subsection{Wall pressure fluctuations}

The results of this paragraph concern the two axial-fans operating in a ducted-flow configuration (see Fig. 2b). In that case there is a radial gap of $1.5 \mathrm{~mm}$ between the pipe and the collar surrounding the fan. It should be also noted that for technical reasons, the ducted experiments have been performed for an angular velocity $\omega=209.5$ rad.s ${ }^{-1}$ (2000 rpm). The presence of the gap substantially reduces the efficiency of the fans without modifying the nominal flow-rate: $F_{A}$ reaches a maximum efficiency $\eta=0.49$ for a flow coefficient $\Phi=\Phi_{N, A}=0.18$. The pressure coefficient is $\Psi=0.15$, slightly lower than in the free-flow experiment which presents no radial gap. The $F_{B}$ fan reaches a maximum efficiency $\eta=0.48$ at a flow coefficient $\Phi=\Phi_{N, B}=0.15$ and a pressure coefficient $\Psi=0.18$. The characteristic curves show similar behavior as in the free-flow case for nominal and overflow-rates. The behaviors at partial flow-rates are completely different owing to the presence of the tube that prevents the flow from being centrifuged. The results reported here are thus limited to flow-rates above $\Phi=0.15$.

The wall pressure fluctuations are compared for two different flow-rates, corresponding to the nominal point of the thin blades fan $\Phi_{N, A}=0.18$ and to the nominal point of the thick blades fan $\Phi_{N, B}=0.15$. In every case, for a fixed diaphragm at the pipe exit, the angular velocity of one of the two fans has been set to $\omega=209.5{\mathrm{rad} . \mathrm{s}^{-1}}^{-1}$ and the angular velocity of the second fan has been adjusted in order to give the same dimensional pressure rise and flow-rate for the two fans.

The squared amplitude spectra of wall pressure at flowrate $\Phi_{N, A}$ are plotted in Figure 7 . The spectra $S(f)$ are expressed in $\mathrm{dB}$ using the formula:

$$
S(f)=20 \log _{10}\left(p^{\prime}(f) / p_{0}\right)
$$

$p^{\prime}(f)$ being the modified Welch-average Fourier Transform of the fluctuating pressure. The sampling time is $10 \mathrm{~s}$, corresponding to approximately 330 fan rotations, and the spectra are an average of eight $50 \%$ overlapping parts, windowed with a Hamming window. The spectra are displayed between 150 and $850 \mathrm{~Hz}$. The reference pressure is $p_{0}=1 \mathrm{~Pa}$.

Both fans exhibit relatively high discrete peaks, corresponding to the blade passing frequency $f_{b p f}(200 \mathrm{~Hz}$ for $F_{A}$ and $202 \mathrm{~Hz}$ for $F_{B}$ ) and its harmonics. The corresponding values are reported in Tab. 2. They are high above the broadband noise. The two fans differ however strongly by the repartition of energy between the different
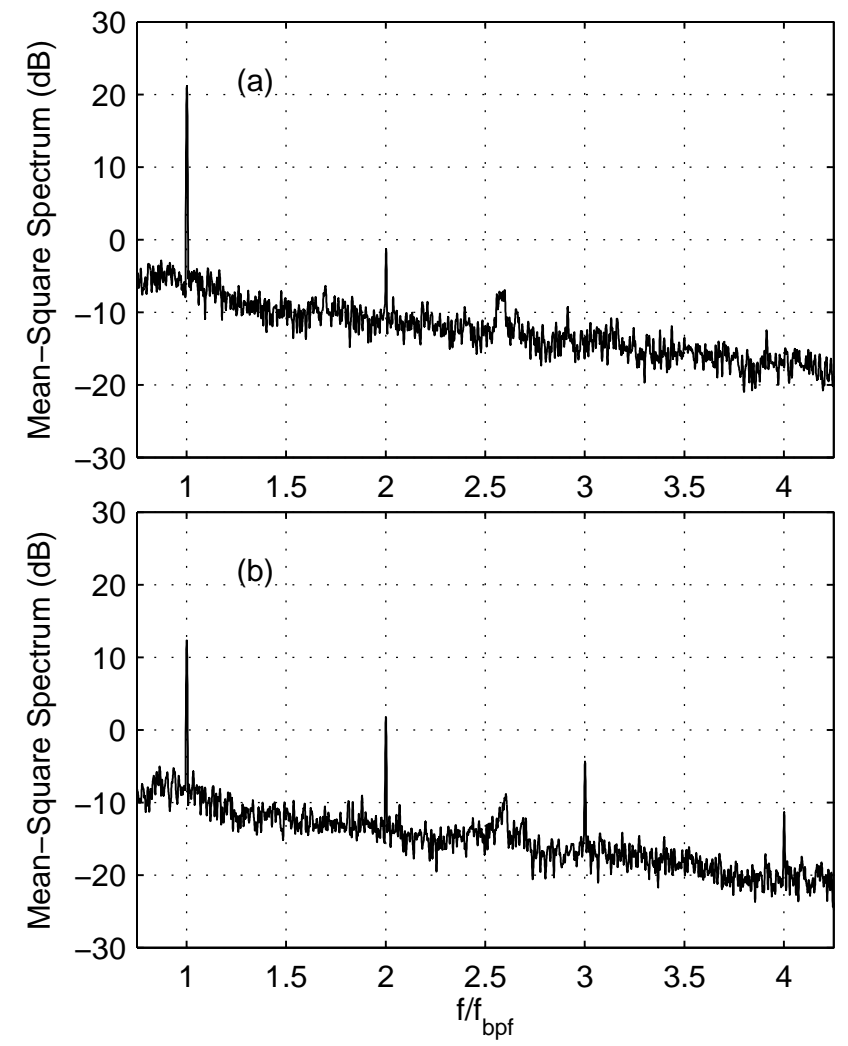

Figure 7: Square amplitude spectra of wall pressure fluctuations for thin blades axial-flow fan $F_{A}$ (a) and thick blades axial-flow fan $F_{B}$ (b) at a flow-rate $\Phi=\Phi_{N}, A=0.18 . F_{A}$ rotates at $\omega=209.5 \mathrm{rad}^{-\mathrm{s}^{-1}}$ and $F_{B}$ rotates at $\omega=211.5$ rad.s $^{-1}$ in order to give the same flowrates and pressure rises.

harmonics. For $F_{A}$, only the fundamental frequency $f_{b p f}$ and the first harmonic $2 f_{b p f}$ are visible. The level at $f_{b p f}$ is $21 \mathrm{~dB}$, and the level at $2 f_{b p f}$ is $-1.5 \mathrm{~dB}$.

For $F_{B}$ fan, the first three harmonics clearly appear. At the blade passing frequency $f_{b p f}$ the level is $12 \mathrm{~dB}$. At $2 f_{b p f}$ it is $2 \mathrm{~dB}$, at $3 f_{b p f}$ it is $-4 \mathrm{~dB}$ and at $4 f_{b p f}$ it is $-11 \mathrm{~dB}$. For this flow-rate, the increase of blades thickness thus seems to lead to a decrease of the first peak level, and to an increase of the harmonics. The global level of fluctuations, i.e. $10 \log _{10}\left(\sigma_{p^{\prime}}^{2} / p_{0}^{2}\right)$, is moreover lower for the thick blades fan $\left(F_{B}: 19 \mathrm{~dB}\right)$ than for the thin blades fan $\left(F_{A}: 23 \mathrm{~dB}\right)$.

The previously described features regarding both the spectral composition and the relative fluctuations levels could be due to the fact that the $F_{A}$ fan is working at its nominal flow-rate while the $F_{B}$ fan is working at overflowrate. To answer this question we have performed the same experiment at the nominal flow-rate of the $F_{B}$ fan.

The squared amplitude spectra of wall pressure at the nominal flow-rate of the thick blades fan $\Phi_{t c k}=0.15$ are plotted in Figure 8 and the levels of the discrete peaks are reported in Tab. 3. The blade passing frequency is now $f_{b p f}=200 \mathrm{~Hz}$ for $F_{B}$ and $193 \mathrm{~Hz}$ for $F_{A}$. Please note that the angular velocity ratio needed to reach the same 

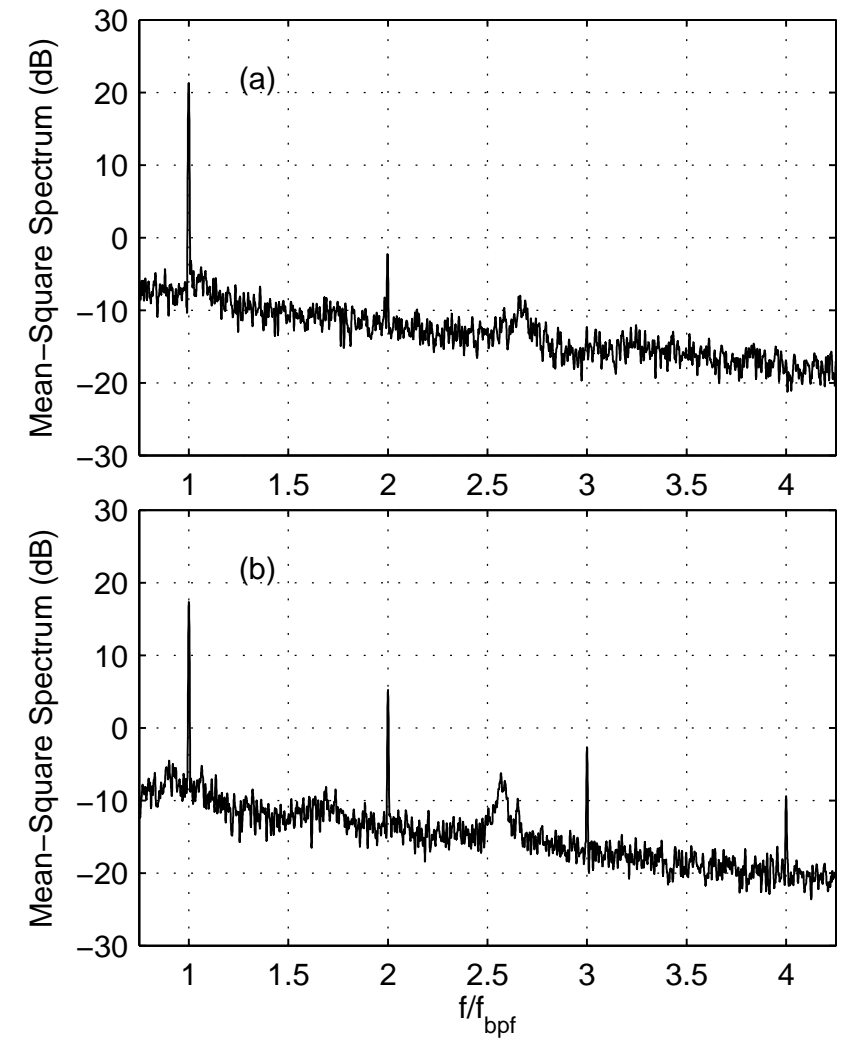

Figure 8: Square amplitude spectra of wall pressure fluctuations for thin blades axial-flow fan $F_{A}$ (a) and thick blades axial-flow fan $F_{B}$ (b) at a flow-rate $\Phi=\Phi_{N, B}=0.15 . \quad F_{B}$ rotates at $\omega=209.5$ rad.s ${ }^{-1}$ and $F_{A}$ rotates at $\omega=202.7$ rad.s ${ }^{-1}$.

dimensional static pressure rise and flow-rate $\omega_{B} / \omega_{A}=$ 1.037 is the same as the ratio discussed in $\S 3.1$ dedicated to the fan overall characteristics.

The results are very similar to the previously described flow-rate: for the $F_{A}$ fan, the fundamental frequency $f_{b p f}$ and the first harmonic $2 f_{b p f}$ are still the only visible peaks in the spectrum. The level at $f_{b p f}$ is $21 \mathrm{~dB}$, and the level at $2 f_{b p f}$ is $-2 \mathrm{~dB}$. For the $F_{B}$ fan, the first three harmonics clearly appear. At the blade passing frequency $f_{b p f}$ the level is $17 \mathrm{~dB}$. At $2 f_{b p f}$ it is $5 \mathrm{~dB}$, at $3 f_{b p f}$ it is $-2.5 \mathrm{~dB}$ and at $4 f_{b p f}$ it is $-9.5 \mathrm{~dB}$. The decrease of the first peak level and the increase of the level of the harmonics is also present at this flow-rate. The global level of fluctuations is $20 \mathrm{~dB}$ for the thick blades fan and $22.5 \mathrm{~dB}$ for the thin blades fan.

\begin{tabular}{c|c|c|c|c|c}
\hline \hline & $f_{b p f}$ & $2 f_{b p f}$ & $3 f_{b p f}$ & $4 f_{b p f}$ & Total \\
\hline$F_{A}$ & 21 & -1.5 & - & - & 23 \\
\hline$F_{B}$ & 12 & 2 & -4 & -11 & 19 \\
\hline \hline
\end{tabular}

Table 2: Level of wall-pressure fluctuations at the blade passing frequency and its harmonics $(\mathrm{dB})$ for both fans at flow-rate $\Phi_{N}, A$.

Figure 9 presents autocorrelation and cross-correlation functions of wall pressure fluctuations for the two fans.

\begin{tabular}{c|c|c|c|c|c}
\hline \hline & $f_{b p f}$ & $2 f_{b p f}$ & $3 f_{b p f}$ & $4 f_{b p f}$ & Total \\
\hline$F_{A}$ & 21 & -2 & - & - & 22.5 \\
\hline$F_{B}$ & 17 & 5 & -2.5 & -9.5 & 20 \\
\hline \hline
\end{tabular}

Table 3: Level of wall-pressure fluctuations at the blade passing frequency and its harmonics $(\mathrm{dB})$ for both fans at flow-rate $\Phi_{N, B}$.

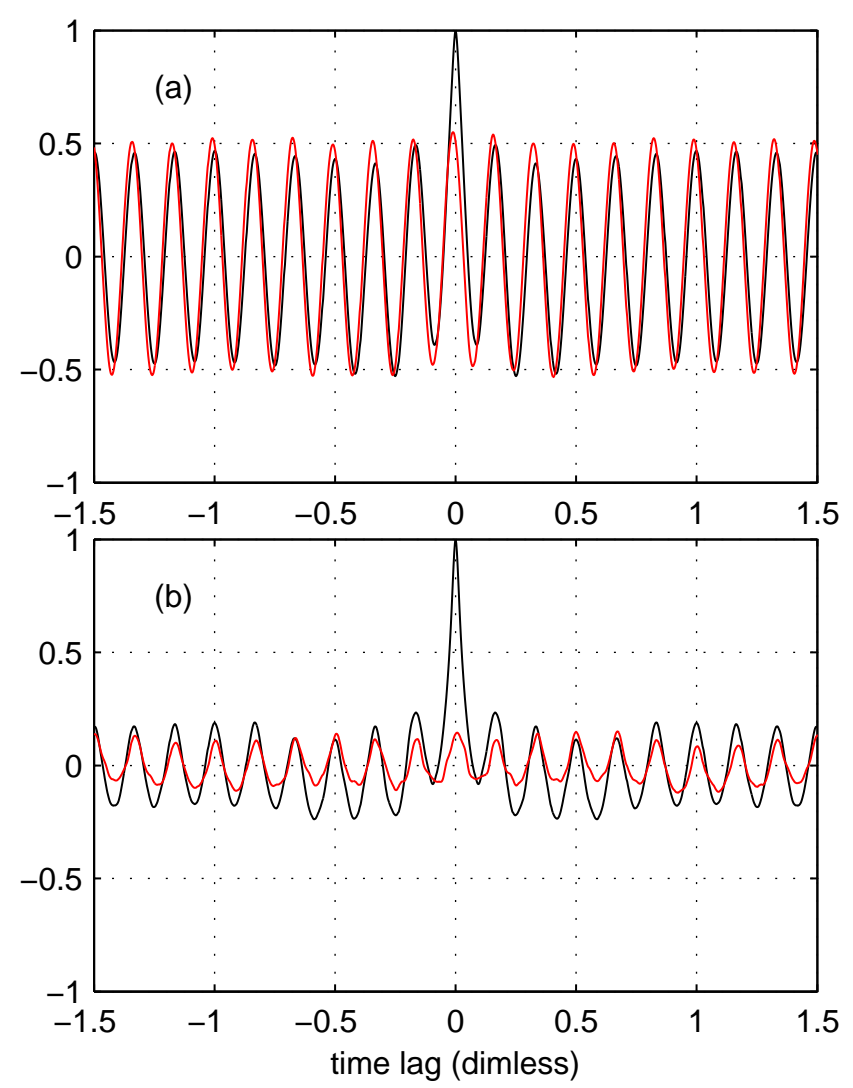

Figure 9: Black curves: autocorrelation function of pressure fluctuations at position 1 (see Fig. 2b). Red curves: cross-correlation function of pressure fluctuations between position 1 and position 5 (see Fig. 2b). Top graph corresponds to $F_{A}$ at $\Phi_{N, A}=0.18$, bottom graph corresponds to $F_{B}$ at $\Phi_{N, B}=0.15$. The time is made non-dimensional with reference to one period of rotation: $t^{\prime}=\frac{t \omega}{2 \pi}$

The black curves correspond to the autocorrelation function:

$$
A(\tau)=\frac{\left\langle p_{1}^{\prime}(t) p_{1}^{\prime}(t-\tau)\right\rangle}{\sigma_{p^{\prime} 1}^{2}}
$$

and the red curves correspond to the cross-correlation function between microphones 1 and 5:

$$
C_{1-5}(\tau)=\frac{\left\langle p_{1}^{\prime}(t) p_{5}^{\prime}(t-\tau)\right\rangle}{\sigma_{p_{1}^{\prime}} \sigma_{p_{5}^{\prime}}}
$$

One can notice that the functions present a periodicity that corresponds to six events in one rotation that is the blade passing frequency. The time lag for the main peak in the cross-correlation function does match the angular distance between the microphones which is one half of a turn here. Another common feature between the two fans is 
that secondary peaks height for the autocorrelation function are close to the peaks height for the cross-correlation function. Some strongly coherent flow structures might be involved in this zone between the rotor and the binding static part. The strong difference between the $F_{A}$ fan and the $F_{B}$ fan is the value of the cross-correlation coefficient. It is significantly lower for the thick blades fan. Local velocity measurements in the wake of the fans may help to better understand this difference and the links between exit-flow dynamics and wall pressure fluctuations.

\subsection{Local velocity measurements in the wake of the fans}

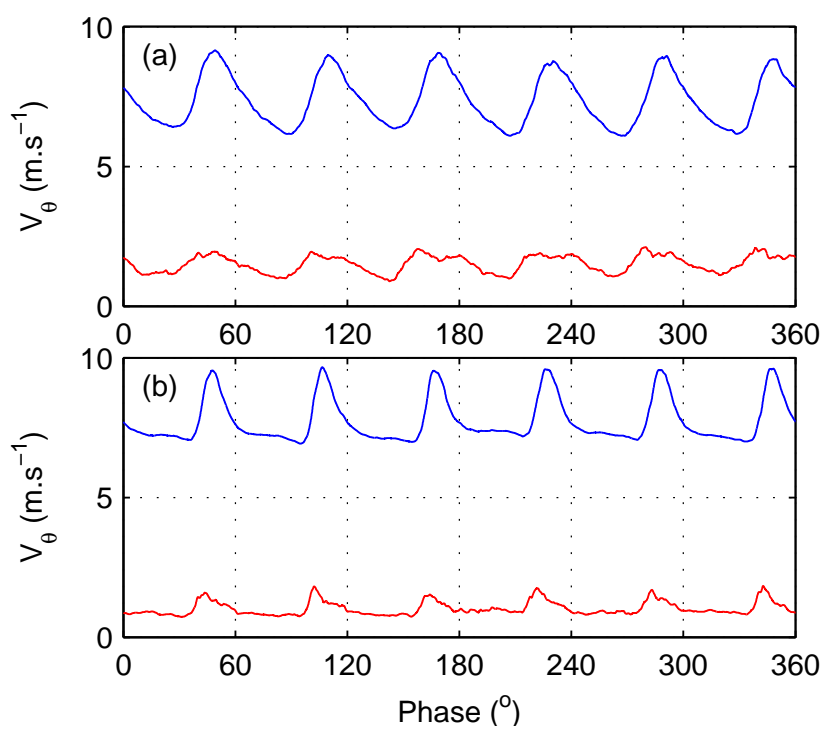

Figure 10: Phase-averaged tangential velocity $V_{\theta}(\phi)$ (blue) and phase-averaged $r m s$ of the tangential velocity $V_{\theta r m s}(\phi)$ (red) at an axial distance $z=24 \mathrm{~mm}$ downstream of the fan and at midspan $r=120 \mathrm{~mm}$. The operating point is close to the nominal point of $F_{A}$. (a): $F_{A}$ rotating at $\omega=205.5$ rad.s ${ }^{-1}$ for a flow-rate $Q=0.620 \mathrm{~m}^{3} \cdot \mathrm{s}^{-1}$ and a pressure rise $\Delta p=125 \mathrm{~Pa}$ corresponding to a flow coefficient $\Phi_{A}=0.178$. (b): $F_{B}$ rotating at $\omega=209$ rad.s ${ }^{-1}$ to give the same flow-rate and pressure rise, that corresponds then to a flow coefficient $\Phi_{B}=0.170$

Time-resolved signals of the tangential velocity downstream of the fans have been measured by Constant Temperature Anemometry (CTA) at an axial distance $z=$ $24 \mathrm{~mm}$ downstream of the fan and at a radius $r=120 \mathrm{~mm}$ that corresponds to mid-span. The rotation rates of the fans have been adjusted to give the same operating point (fixed diaphragm at the inlet of the free-flow experimental facility, flow-rate $Q$ and pressure rise $\Delta p)$. This point is close to the nominal flow-rate of $F_{A}\left(\Phi_{N, A}=0.18\right)$ and the fans rotate at an angular velocity close to $\omega=$ 209 rad.s ${ }^{-1}$ (2000 rpm). An amount of $2^{19}$ samples have been recorded at an acquisition rate of $120 \mathrm{kHz}$. That corresponds to roughly 150 revolutions of the fans and to approximately 3600 samples per revolution. The rotation rates have been measured with an encoder, synchronized to the CTA data acquisition, in order to perform analysis of phase-averaged signals [2]. This point has been chosen
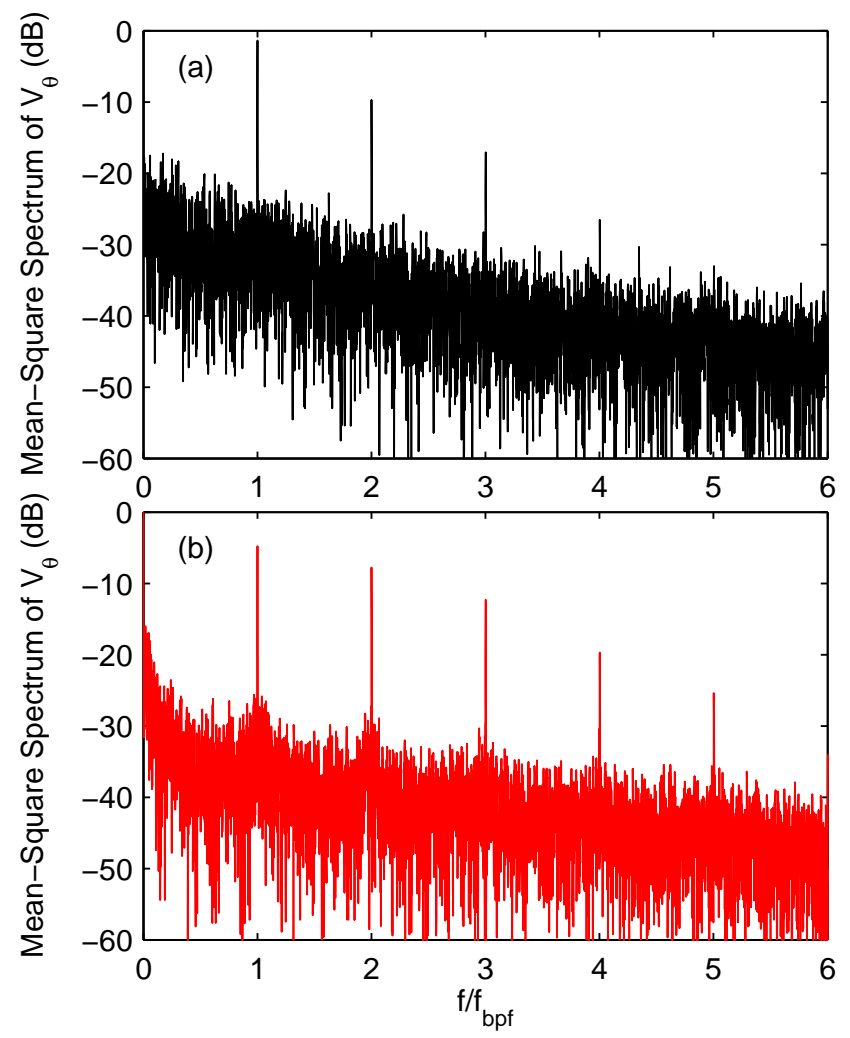

Figure 11: Square amplitude spectra of $V_{\theta}(t)$ under the conditions of Fig. 10. (a): $F_{A}$ fan. (b): $F_{B}$ fan

as close as possible to the fans blades, and such that the three components of the velocity are close for both fans, as can be seen in Fig. 6 .

The phase-averaged signals of the tangential velocity $V_{\theta}(\phi)$ are plotted in Fig. 10, $\phi$ being the phase on one revolution, in degree. Portions of signal of length corresponding to one revolution have been averaged. The signals for $F_{A}$ (Fig. 10a) and for $F_{B}$ (Fig. 10b) are periodic with a periodicity of $60^{\circ}$ that corresponds to the blade passages. The shape of the signal for each blade is very similar owing to the very good symmetry and alignment of the system. The mean velocity is respectively $7.4 \mathrm{~m} . \mathrm{s}^{-1}$ for $F_{A}$ and $7.7 \mathrm{~m} . \mathrm{s}^{-1}$ for $F_{B}$. For $F_{A}$, the signal has a more regular shape than for the thick blades fan $F_{B}$, and the peakto-peak amplitude is bigger. These peak-to-peak phaseaveraged fluctuations give the biggest contribution to the total standard deviation of the velocity. They should also give the biggest contribution to the peaks at blade passing frequency and its harmonics in velocity spectra as reported by Kergoulay et al. [2] and as can be seen from the phaseaveraged $r m s$ of the tangential velocity $V_{\theta r m s}(\phi)$ plotted in red in Fig. 10.

The local tangential velocity spectra are plotted in Fig. 11. The peaks at the blade passing frequency and its harmonics are clearly visible and are reported in Tab. 4. The same features as for the wall pressure fluctuations studied in $\S 4.1$ are present: $F_{A}$ has a stronger peak at 
$f_{b p f}$ but smaller and fewer secondary peaks. This is consistent with the shape of the phase-averaged velocity signals (Fig. 10) and the fact that the main contribution to the peaks arises from this phase-averaged component of the velocity signal.

\begin{tabular}{c|c|c|c|c|c}
\hline \hline & $f_{b p f}$ & $2 f_{b p f}$ & $3 f_{b p f}$ & $4 f_{b p f}$ & $5 f_{b p f}$ \\
\hline$F_{A}$ & -1.4 & -9.7 & -17.1 & -26.5 & - \\
\hline$F_{B}$ & -4.8 & -7.8 & -12.3 & -19.7 & -25.4 \\
\hline \hline
\end{tabular}

Table 4: Level of tangential velocity fluctuations at the blade passing frequency and its harmonics $(\mathrm{dB})$ for both fans close to flow-rate $\Phi_{N, A}$

\section{Conclusions}

Two fans that differ only in the thickness of their blades were studied in order to highlight the effects of thickness on overall performances and the pressure and velocity fluctuations that are generated. The first fan, which has very thin blades, was designed to be part of the cooling system of an automotive vehicle power unit. The second compatible with the rotomoulding conception process, that generates only hollow parts with large edge radius, has much thicker blades.

The performances of the fans were measured and the results indicate that the overall performances were substantially equivalent, with a drop of $8 \%$ of pressure rise at conception flow-rate for the thick blades fan and a maximum efficiency that is $3 \%$ lower than the efficiency of the thin blades fan and that is shifted towards lower flow-rates.

Contrary to what one could a priori think the thick blades fan does present a certain number of interesting features:

1. The overall performances are only slightly decreased. The conception point can be reached by a relative increase of rotational speed of $4 \%$ for the same power consumption. The fact that the characteristic curve is steeper could be positive: the flow-rate is more stable with respect to small variations of the circuit hydraulic impedance. Finally, at very partial flowrates, the thick blades fan produces more pressure rise than the thin blades fan, which could be beneficial concerning stall instabilities.

2. The mean exit-flow fields present significant diffferences. The tangential velocity component is stronger and remains high further away downstream of the thick blades fan $F_{B}$, which may increase the efficiency of a potential stator. The exit-flow moreover remains axial on a wider operating range. The thin blades fan $F_{A}$ leads to a more centrifuged discharging flow, which may be a configuration of interest in some applications, for instance for automotive cooling with the fan sucking air through the heat exchanger.
3. The wall pressure fluctuations just downstream of the fan are lower for the thick blades and their spectral decomposition is completely different. The same features are observed on velocity fluctuations. The spectral decomposition that shows stronger peaks at the blade passing frequency and less harmonics for the thin blades fan than for the thick blades fan, together with the phase-averaged analysis of velocity signals suggest that the pressure fluctuations are due to strong coherent wakes that are more dissipated with the use of thick blades. These measurements will be used as a benchmark for high-order finite volume CFD codes developed for aeroacoustics in the laboratory [15], and data are also available on request.

Further numerical and experimental investigations are planed to better understand the origins of the observed features that are reported in the present Article. The open questions that remain concern the influence of the blade thickness and of its distribution from leading edge to trailing edge on the blade loading and how this affects the efficiency and shift of the nominal flow-rate, the centrifugation of the exit-flow and the modifications of the pressure and velocity spectra.

\section{References}

[1] T. Fukano, Y. Kodama, and Y. Takamatsu. Noise generated by low pressure axial flow fans, iii: Effects of rotational frequency, blade thickness and outer blade profile. Journal of Sound and Vibration, 56:261-277, 1978

[2] G. Kergourlay, S. Kouidri, G. W. Rankin, and R. Rey. Experimental investigation of the $3 \mathrm{~d}$ unsteady flow field downstream of axial fans. Flow Measurement and Instrumentation, 17:303314, 2006.

[3] J. Vad, A. R. A. Kwedikha, Cs. Horváth, M. Balczó, M. M. Lohász, and T. Régert. Aerodynamic effects of forward blade skew in axial flow rotors of controlled vortex design. Proceedings of the Institution of Mechanical Engineers, Part A: Journal of Power and Energy, 221:1011-1023, 2007.

[4] J. Hurault, S. Kouidri, F. Bakir, and R. Rey. Experimental and numerical analysis of the sweep effect on three-dimensional flow downstream of axial flow fans. Flow measurement and instrumentation, 21:155-165, 2010.

[5] M. Anandha Rao and J. L. Throne. Principles of rotational molding. Polymer Engineering \& Science, 12:237-264, 1972.

[6] A. Tcharkhtchi and J. Verdu. Structure-processibility relationships during rotational moulding of plastics. Advanced Engineering Materials, 6:983-992, 2004.

[7] B. Thwaites. Incompressible Aerodynamics, An Account of the Theory and Observation of the Steady Flow of incompressible Fluid past Aerofoils, Wings, and Other Bobies. Dover Publication, Inc., 1987.

[8] S. F. Hoerner. Fluid-Dynamic Lift. Hoerner Fluid Dynamics, 1985.

[9] R. J. Roelke and J. E. Haas. The effect of rotor blade thickness and surface finish on the performance of a small axial flow turbine. Journal of Engineering for Power, 105:377-382, 1983.

[10] J. Estevadeordal, S. Gorgineni, W. Copenhaver, G. Bloch, and M. Brendel. Flow field in a low-speed axial fan: a dpiv investigation. Experimental Thermal Fluid Science, 23:11-21, 2000.

[11] C. Sarraf, H. Djeridi, S. Prothin, and J.Y. Billard. Thickness effect of naca foils on hydrodynamic global parameters, bound- 
ary layer states and stall establishment. Journal of Fluids and Structures, 26:559-578, 2010.

[12] K.-S. Lee, K.-Y. Kim, and A. Samad. Design optimization of low-speed axial flow fan blade with three-dimensional RANS analysis. Journal of Mechanical Science and Technology, 22:1864-1869, 2008.

[13] R. Noguera, R. Rey, F. Massouh, F. Bakir, and S. Kouidri. Design and analysis of axial pumps. In ASME Fluids Engineering, Second Pumping Machinery Symposium, Washington, USA., pages 95-111, 1993.

[14] J. Hurault, S. Koudri, F. Bakir, and R. Rey. Experimental and numerical investigation of downstream turbulence in axial flow fan. In Fan Noise 2007 International Symposium, Lyon, 2007.

[15] F. Ravelet, S. Khelladi, H. Nouri, F. Bakir, H. Kim, Y. Bae, and Y. J. Moon. Study of the aerodynamics/aeroacoustics of an axial-flow fan: Experimental validation of a les/lpce/brinkman penalization method. In $16^{t} h$ AIAA/CEAS Aeroacoustics Conference, 7-9 June 2010, Stockholm, Sweden., pages AIAA 2010$3869,2010$. 